\title{
The Virtual Patient and Family Advisory Council in the COVID-19 Era
}

\author{
Jeffrey D. Schlaudecker, MD, MEd and Keesha Goodnow, BAE
}

Background: In 2016, we launched our first Patient and Family Advisory Council (PFAC) as a means of collaborating with our patients and families to improve care. Using an Internet-based remote meeting technology, we transitioned to a virtual platform in April.

Methods: We have conducted 12 PFAC meetings across 4 sites to date. Virtual PFAC meeting topics over the past few months include communication about the coronavirus, community resources needed by patients during the pandemic, telehealth visit troubleshooting, current office policy, and changing work flow. A convenience sample of advisors generated qualitative responses on the transition from inperson meetings to a virtual platform.

Results: Attendance increased as we transitioned to a virtual platform from 13.2 advisors to 14.7 advisors. Advisors affirm the value of a PFAC and importance of patient engagement, especially during this pandemic. Patient advisors confirm the role of patient voice in pandemic-induced practice changes.

Discussion: The transition of our PFACs to a virtual platform continues to generate critically important partnerships between patients and providers. In this time of health care uncertainty and stress for patients, providers, and staff, this partnership remains our most valuable asset.

Conclusion: Patient voice provides reliable and relevant information for practices through virtual PFAC meetings. (J Am Board Fam Med 2021;34:S37-S39.)

Keywords: COVID-19, Family Medicine, Pandemics, Patient-Centered Care, Patient Participation, Quality Improvement, Telemedicine

During this global pandemic of COVID-19, our US health care system has experienced unprecedented shifts in remarkably short time spans. ${ }^{1}$ Long-standing traditions and regulations around telehealth, the Health Insurance Portability and Accountability Act, and face-to-face requirements were quickly waived, ${ }^{2}$ and providers and patients alike had to transition almost overnight to a system that met the new reality of stay-at-home orders and videoconferencing. As the need for primary care,

This article was externally peer reviewed.

Submitted 26 August 2020; revised 21 October 2020; accepted 26 October 2020.

From the University of Cincinnati, College of Medicine, Department of Family and Community Medicine, Cincinnati, $\mathrm{OH}$ (JDS, KG); Christ Hospital/University of Cincinnati Family Medicine Residency, Cincinnati, OH (JDS).

Funding: This work was supported by the Health Resources \& Services Administration Primary Care Training and Enhancement Grant T0BHP28567: Partnering with Underserved Patients: A Novel Health Transformation Curriculum.

Conflicts of interest: None.

Corresponding author: Keesha Goodnow BAE, University of Cincinnati, College of Medicine, 231 Albert Sabin Way, MSB 4353B, Cincinnati, OH 45267-0582 (E-mail: keesha. goodnow@uc.edu). well-child checks, and medication management for chronic diseases continued, so did practices' need to authentically partner with patients and families.

In 2016, we launched our first Patient and Family Advisory Council (PFAC) as a means of collaborating with our patients and families to improve care. $^{3}$ The success of this council led to expansion at 3 additional community underserved partner sites. As a result of the pandemic, our offices' monthly PFAC meetings could no longer meet face to face, but our need for patient and family input has never been greater. Using an Internet-based remote meeting technology, we transitioned to a virtual platform in April. We have conducted 12 PFAC meetings across 4 sites to date.

One of our PFACs operates at a residency-based family medicine center (FMC). This FMC is located on the campus of an urban, community teaching hospital. Nine faculty physicians and 26 resident physicians provide full-spectrum family medicine for approximately 7000 patients, with 23,000 annual visits in a practice that is $60 \%$ Medicare/Medicaid. At this site, we have 31 advisors, which include 15 
patient and family advisors, 5 staff advisors, 9 resident physician advisors, and 2 faculty physician advisors. Having a diverse and representative group of advisors has been a primary goal since the creation of the PFAC 5 years ago. Our current membership of 15 patients represents an inclusive and representative cohort of our practice.

Our PFAC meetings provide an opportunity for advisors to share meaningful experiences and serve as a vehicle for 360-degree feedback to the clinic. The 3 virtual PFAC meetings focused on a combination of clinic-identified and advisor-driven content. This included PFAC feedback on wording of communication regarding COVID-19, modes of sharing information with patients and families during the pandemic, patient and physician personal experience with telehealth visits, modified office policy, and patient flow adjustments to preserve social distancing. In addition, an expansive community resource guide was compiled by faculty physicians in the office. PFAC advisors made recommendations about which resources were most important, relevant, and accessible to support patients, families, and the broader community during this difficult time (Figure 1).

Participation and commitment are challenges for many PFACs. A surprising outcome of transitioning to a virtual platform was attendance, increasing from 13.2 advisors to 14.7 advisors per meeting. A virtual PFAC meeting removed barriers of transportation, travel time, and childcare needs. To promote online success, a how-to guide was sent to educate advisors on using the Internet-based remote meeting technology. For those requiring more support, one-on-one training was provided via a premeeting with the PFAC coordinator.

The value of these meetings is difficult to quantify but is perhaps best captured in the voices of our advisors. The following quotes were obtained from a convenience sample of advisors from 4 openended questions sent by e-mail to the PFAC membership: (1) Are patient and family advisors still needed in the era of telehealth? (2) What is the role of a PFAC for a virtual office? (3) What can a PFAC do to help patients and practices in the era of telehealth and virtual offices? (4) How does a PFAC add value in times of uncertainty, like a pandemic? In their words, the impact of our PFAC is clear:

A Patient and Family Advisory Council is needed more than ever! As things are changing, our perspective and input is key. Our role is the same as it's always been-come up with unique solutions to frustrations and problems. PFAC members can help smooth out bumps and figure out problems. It makes me happy that a doctor's office cares enough to enlist our PFAC to help fix and work through things. In these new and uncertain times, it is comforting to know that patients have someone from their perspective having input in these delicate changes.-Patient Advisor

The PFAC is a great way to take the pulse of the group of patients and how they are approaching their own health during this time. It is a scary time and people need encouragement to go to the doctor and utilize all they have to offer. When sometimes it is hard to determine what to

Figure 1. Overview of transition to virtual PFAC. Abbreviation: PFAC, Patient and Family Advisory Council.

\section{Overview of transition to virtual PFAC}

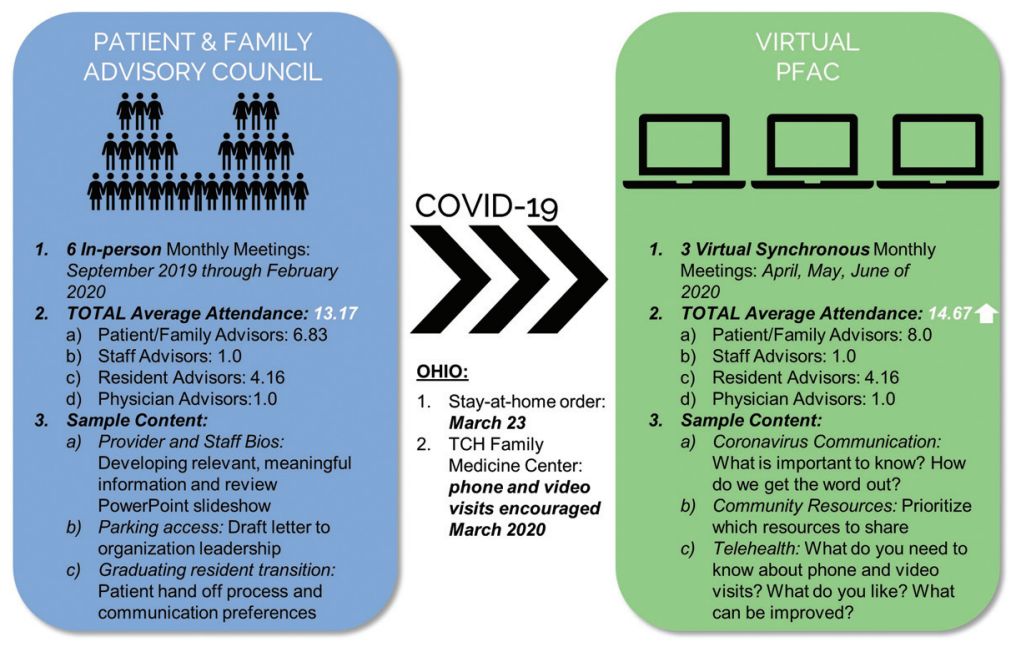


believe from the media and social media, I see the PFAC leading the way to increase communication and help ease fears during these uncertain times.-Patient Advisor

Advisors are definitely needed with the changes in how patients are cared for in the current environment. The virtual and telephone component needs the opinion of advisors to improve the way patients are handled and to keep them confident that they are receiving the appropriate care and advice. A PFAC adds value by providing transparency to total patient care.-Patient Advisor

In a virtual office, the role of the PFAC is for each member to look a little deeper. Potentially asking ourselves some hard questions. Is the practice allinclusive in the methods and forms of virtual communication and care? An engaged PFAC can share their experience of identifying the problem, what was tried, ideas or barriers that were identified and the solution that was deemed best.-Staff Advisor

A PFAC can play a key role in reviewing how to adjust our delivery of care to meet patients where they are and address challenges and barriers to telehealth. A PFAC that meets virtually can provide a sense of hope to the community and patient population it serves. It can provide a level of comfort as a group of patients and staff still meeting and focused solely on practice improvements.-Staff Advisor

More than ever the PFAC is needed in the era of telehealth. When patients are no longer in the office, we lose some of the human connectivity we rely on as physicians to form those relationships that are so key to a successful therapeutic relationship. With that diminished, we need every avenue possible to engage the patients in our office, partly for them to know we are still here but also so we can hear of their troubles, struggles, success etc.Family Medicine Resident Physician Advisor

The value of a PFAC is very clear during a pandemic. Hearing patient and family concerns and points of confusion is useful. It helps us stay focused on where patients and families need more help or education. When times are uncertain, the PFAC is all the more useful to ensure better teamwork and communication.-Faculty Physician Advisor

The transition of our PFACs to a virtual platform continues to generate critically important partnerships between patients and providers. A virtual platform also may have decreased some barriers to attendance, enabling a larger cohort. In our current time of health care uncertainty and the stress of a global pandemic, the partnership implicit within our PFAC remains our most valuable asset. Engaging patient and family advisors in the response to COVID-19 is critical in providing meaningful and important information, safeguarding patient safety, and promoting essential community support and outreach. ${ }^{4}$ During these dramatic changes, patient voice remains constant in providing reliable and relevant information for our FMC practice through virtual PFAC meetings.

To see this article online, please go to: http://jabfm.org/content/ 34/Supplement/S37.full.

\section{References}

1. Gostin LO, Friedman EA, Wetter SA. Responding to Covid-19: how to navigate a public health emergency legally and ethically. Hastings Cent Rep 2020;50:8-12.

2. Centers for Medicare and Medicaid Services. Medicare telemedicine health care provider fact sheet. 2020. Available from: https:/www.cms.gov/newsroom/factsheets/medicare-telemedicine-health-care-providerfact-sheet. Accessed May 30, 2020.

3. Schlaudecker JD, Goodnow K, Goroncy A, et al. Meaningful partnerships: stages of development of a Patient and Family Advisory Council at a family medicine residency clinic. J Particip Med 2019;11: e12105. doi:10.2196/12105.

4. Institute for Patient- and Family-Centered Care. COVID-19 and patient- and family-centered care frequently asked questions. 2020. Available from: https:// www.ipfcc.org/bestpractices/covid-19/IPFCC_PFCC_ and_COVID.pdf. Accessed May 30, 2020. 